\title{
Mild Cognitive Impairment Subtypes Are Associated With Peculiar Gait Patterns in Parkinson's Disease
}

\begin{abstract}
Marianna Amboni ${ }^{1,2 *}$, Carlo Ricciardi ${ }^{3,4}$, Sofia Cuoco ${ }^{1}$, Leandro Donisi ${ }^{4,5}$, Antonio Volzone ${ }^{1}$, Gianluca Ricciardelli ${ }^{6}$, Maria Teresa Pellecchia ${ }^{1}$, Gabriella Santangelo7, Mario Cesarelli, ${ }^{3,4}$ and Paolo Barone ${ }^{1}$
\end{abstract}

\begin{abstract}
1 Department of Medicine, Surgery and Dentistry, Center for Neurodegenerative Diseases (CEMAND), University of Salerno, Fisciano, Italy, ${ }^{2}$ Istituto di Diagnosi e Cura (IDC) Hermitage-Capodimonte, Naples, Italy, ${ }^{3}$ Department of Electrical Engineering and Information Technology, University of Naples Federico II, Naples, Italy, ${ }^{4}$ Istituti Clinici Scientifici Maugeri Istituto di Ricovero e Cura a Carattere Scientifico (IRCCS), Telese Terme, Italy, ${ }^{5}$ Department of Advanced Biomedical Sciences, University of Naples Federico II, Naples, Italy, ${ }^{6}$ Department of Medicine, Azienda Ospedaliera Universitaria OO. RR. San Giovanni di Dio e Ruggi D'Aragona, Salerno, Italy, ${ }^{7}$ Department of Psychology, University of Campania Luigi Vanvitelli, Caserta, Italy
\end{abstract}

OPEN ACCESS

Edited by:

Woon-Man Kung,

Chinese Culture University, Taiwan

Reviewed by:

Saul Martinez-Horta,

Hospital de la Santa Creu i Sant Pau,

Spain

Davide Maria Cammisuli,

University of Milan, Italy

Daniel Collerton,

Newcastle University, United Kingdom

*Correspondence: Marianna Amboni mamboni@unisa.it

Specialty section: This article was submitted to

Parkinson's Disease and Aging-related Movement

Disorders,

a section of the journa

Frontiers in Aging Neuroscience

Received: 22 September 2021 Accepted: 31 January 2022 Published: 01 March 2022

Citation:

Amboni M, Ricciardi C, Cuoco S, Donisi L, Volzone A, Ricciardelli G, Pellecchia MT, Santangelo G, Cesarelli M and Barone P (2022) Mild Cognitive Impairment Subtypes Are Associated With Peculiar Gait

Patterns in Parkinson's Disease.

Front. Aging Neurosci. 14:781480.

doi: 10.3389/fnagi.2022.781480
Background: Mild cognitive impairment $(\mathrm{MCl})$ is frequent in Parkinson's disease (PD) and represents a risk factor for the development of dementia associated with PD (PDD). Since PDD has been associated with disability, caregiver burden, and an increase in health-related costs, early detection of $\mathrm{MCl}$ associated with $\mathrm{PD}$ (PD-MCl) and its biomarkers is crucial.

Objective: Given that gait is considered a surrogate marker for cognitive decline in $\mathrm{PD}$, the aim of this study was to compare gait patterns in PD-MCl subtypes in order to verify the existence of an association between specific gait features and particular $\mathrm{MCl}$ subtypes.

Methods: A total of 67 patients with PD were consecutively enrolled and assessed by an extensive clinical and cognitive examination. Based on the neuropsychological examination, patients were diagnosed as patients with $\mathrm{MCl}(\mathrm{PD}-\mathrm{MCl})$ and without $\mathrm{MCl}$ (no-PD-MCl) and categorized in $\mathrm{MCl}$ subtypes. All patients were evaluated using a motion capture system of a BTS Bioengineering equipped with six IR digital cameras. Gait of the patients was assessed in the ON-state under three different tasks (a single task and two dual tasks). Statistical analysis included the $t$-test, the Kruskal-Wallis test with post hoc analysis, and the exploratory correlation analysis.

Results: Gait pattern was poorer in PD-MCI vs. no-PD-MCl in all tasks. Among PD-MCl subtypes, multiple-domain PD-MCl and amnestic PD-MCl were coupled with worse gait patterns, notably in the dual task.

Conclusion: Both the magnitude of cognitive impairment and the presence of memory dysfunction are associated with increased measures of dynamic unbalance, especially in dual-task conditions, likely mirroring the progressive involvement of posterior cortical networks.

Keywords: $\mathrm{MCl}$ (mild cognitive impairment), Parkinsion's disease (PD), gait analysis, gait pattern characteristics, cognitive decline 


\section{INTRODUCTION}

Cognitive decline is frequent in Parkinson's disease (PD) even in the early stages, and it occurs as a dysfunction in executive, attention, memory, language, and visuospatial domains (Barone et al., 2011). The full spectrum of cognitive skills in PD spans from normal cognition, through mild cognitive impairment (PDMCI), to PD dementia (PDD) (Aarsland et al., 2017). A recent meta-analysis has shown that PD-MCI has a pooled prevalence of $40 \%$, which is associated with older age, lower education, longer disease duration, higher levodopa equivalent daily dose, more severe motor symptoms, postural instability/gait difficulty motor subtype, poorer quality of life, and higher levels of apathy and depression (Baiano et al., 2020). In addition, PD-MCI represents a risk factor for the development of PDD (Hoogland et al., 2017; Saredakis et al., 2019), with a higher rate of conversion to dementia in the amnestic PD-MCI subtype relative to the nonamnestic PD-MCI subtype (Chung et al., 2019). Since PDD has been associated with increased disability, caregiver burden, and risk for institutionalization with a consequent increase in healthrelated costs (Svenningsson et al., 2012), the early detection of MCI and its biomarkers is crucial for the identification of a PD subpopulation at a higher risk of worse disease progression (Mollenhauer et al., 2014).

Cognition and gait in PD appear to be closely related in complex ways. Gait is no longer considered merely an automated motor task but an activity requiring multiple cognitive skills, ensuring safe mobility (Amboni et al., 2013). In a previous study, we have shown that dysfunctions in specific gait parameters that are poorly responsive to levodopa and are highly sensitive to dualtask conditions are associated with PD-MCI, and visuospatial impairment is strongly associated with instability in patients with PD (Amboni et al., 2012). On the one hand, these findings are in line with the hypothesis that dopa-resistant gait components and cognitive dysfunction might share common non-dopaminergic network dysfunction (Nonnekes et al., 2016); on the other hand, they are consistent with the recent findings that balance control would rely on posterior cortical networks (Morris et al., 2019). Moreover, we have provided insight into the chronological relationship between gait and cognitive decline in patients with PD, by showing that step length during a cognitive task on medication predicts subsequent executive/attention dysfunction (Amboni et al., 2018).

More recently, several studies have focused on the ability of gait analysis to distinguish subjects with $\mathrm{MCI}$ and different types of dementias. In particular, Mc Ardle et al. (2020) have shown that wearable sensors-based gait analysis was able to identify subjects with MCI, dementia with Lewy bodies (DLB), Alzheimer's disease (AD), and PDD (Mc Ardle et al., 2020), whereas de Oliveira Silva et al. (2020) could distinct healthy controls and also patients with $\mathrm{MCI}$ and $\mathrm{AD}$ by using a videogrammetry system (de Oliveira Silva et al., 2020). In addition, gait-based machine learning approaches were able to discriminate different types of MCI, such as patients with PDMCI and non-PD-MCI (Chen et al., 2020) or to detect the presence of MCI in PD (Ricciardi et al., 2020). Finally, Xie et al. (2019) employed an inertial-sensor-based wearable instrument to distinguish patients with amnestic $\mathrm{MCI}$ and cognitively normal subjects.

At present, studies comparing quantitative gait measures in PD-MCI subtypes are lacking. The main aim of this study was to compare gait patterns in PD-MCI subtypes in order to verify the existence of an association between specific gait features and particular MCI subtypes. This could shed further light both on the neurobiological substrate of different neuropsychological profiles and on the relationship between gait and cognition in PD.

\section{MATERIALS AND METHODS}

\section{Study Design and Population}

The study sample consisted of 67 patients with PD, consecutively enrolled between February 2018 and June 2021. Participants were selected from patients referred to the Movement Disorders Unit of the Institute for Diagnosis and Care Hermitage-Capodimonte of Naples and the Center for Neurodegenerative Diseases of the University of Salerno. All patients fulfilled the Movement Disorder Society (MDS) clinical diagnostic criteria for PD (Postuma et al., 2015). The inclusion criteria were as follows: age $\geq 45$ years; Hoehn and Yahr $(\mathrm{H} \& \mathrm{Y})$ score $\leq 3$; disease duration < 10 years; and antiparkinsonian treatment at a stable dosage during the previous 4 weeks. The exclusion criteria were as follows: gait requiring assistance; dementia according to the clinical diagnostic criteria for PDD (Emre et al., 2007); clinically significant comorbidities, including other neurological disorders, orthopedic diseases, or cardiovascular/respiratory diseases; anticholinergic or neuroleptic treatment; and brain surgery.

\section{Standard Protocol Approvals, Registrations, and Patient Consent}

This study was performed in accordance with the 1964 Declaration of Helsinki and was approved by Campania Sud, the reference ethics committee of the Center for Neurodegenerative Diseases of the University of Salerno. Written informed consent was obtained from all subjects.

\section{Clinical and Cognitive Evaluations}

All subjects were evaluated using a detailed assessment that included demographic, clinical, and anthropometric data. In addition, they completed the Italian version of the Montreal Cognitive Assessment (MoCA) and an extensive neuropsychological battery comprising the following tests: (1) the Rey Auditory 15-word Learning Test, immediate recall and delayed recall, and the Rey-Osterrieth complex figure delayed recall for the memory domain (Caltagirone et al., 1979; Caffarra et al., 2002); (2) the Stroop Color-Word Test and the Trail Making Test B-A for the attention domain (Giovagnoli et al., 1996; Barbarotto et al., 1998); (3) Phonological verbal fluency and the Clock Drawing Test for the executive domain (Caltagirone et al., 1979; Siciliano et al., 2016); (4) the Benton's Judgment of Line Orientation and the Constructional apraxia test for the visuospatial domain (Spinnler and Tognoni, 1987; Ferracuti et al., 2000); and (5) Actions and objects naming tests for the language domain (Capasso and Miceli, 2001). 
The test scores were corrected for current normative values. All neuropsychological tests were administered to the patients during the pharmacological ON-state. The diagnosis of PDMCI by level II category and PD-MCI subtyping were based on the neuropsychological assessment according to the Movement Disorders Society Task Force guidelines (Litvan et al., 2012).

\section{Gait Analysis}

All patients underwent gait analysis through a BTS Bioengineering system. The SMART DX is an optical system equipped with six IR cameras, two video cameras, two force plates, a set of passive markers, and an elaborator. The Davis protocol was used for all subjects, comprising the following four phases (Davis et al., 1991):

1. Collection of anthropometric measures of the patient (height, weight, and leg length).

2. Positioning of 22 reflective markers on specific points of the body of the patient.

3. The standing phase, consists of acquiring the patient while standing up on the force plate.

4. The walking phase on a straight path of $10 \mathrm{~m}$ during three different tasks including one single task and two dual tasks, namely:

a. GAIT: normal gait (single task).

b. MOT: walking while carrying a tray with two glasses filled with water (dual task).

c. COG: walking while serial subtracting $7 \mathrm{~s}$ starting from 100 (dual task).

Each task was performed four times.

Prior to commencing the trials, all participants were trained to walk at a normal pace at their usual speed, without any instructions to prioritize walking or performing the concomitant task. This procedure generated a report from which spatial and temporal parameters were extracted.

All participants were evaluated in the self-defined best "ONstate" while receiving their typical dopaminergic drugs.

\section{Statistical Analysis}

The statistical analysis was conducted by using Statistical Package for Social Science (SPSS, version 25). First, spatial and temporal variables of gait analysis were compared between patients with PD-MCI and with no-PD-MCI; then, they were compared among patients with no-PD-MCI, with single-domain PD-MCI, and with multiple-domain PD-MCI; and finally, they were compared among patients with no-PD-MCI, with non-amnestic PD-MCI, and with amnestic PD-MCI.

The $t$-test was used to compare two groups; since the analyzed samples contained more than 30 subjects, a normal distribution of the data is fair. Whereas, to compare three groups, given the small sample size for each group, the Kruskal-Wallis test was applied, and when significant, subsequent appropriate posthoc tests were used in order to make the pairwise comparisons, thus correcting the $p$-values for multiple comparisons. Finally, an exploratory correlation study through the coefficient of
Spearman was performed between spatiotemporal parameters and neuropsychological test scores.

Alpha significance level was set to $p<0.05$ for all statistical analyses.

\section{RESULTS}

Thirty-two out of 67 patients were diagnosed as PD-MCI and 35 patients as no-PD-MCI. The 2 groups did not significantly differ on demographic and anthropometric variables, but they showed a trend toward significance on age $(p=0.053)$. When comparing clinical variables, the two groups differed significantly on the Movement Disorder Society Unified Parkinson's Disease Rating Scale (MDS-UPDRS) part III $(p=0.014)$ and, consequently, showed a trend toward significance on total MDS-UPDRS $(p=0.050)$ (Table 1).

\section{Parkinson's Disease-Mild Cognitive Impairment vs. No-Parkinson's Disease-Mild Cognitive Impairment}

Statistical analyses comparing all spatial and temporal gait parameters between patients with no-PD-MCI and PD-MCI are presented in Table 2 .

In all three tasks, patients with PD-MCI exhibited poorer gait patterns when compared with patients with no-PD-MCI. In comparison with patients with no-PD-MCI, patients with PD-MCI exhibited shortened cycle and step length in all tasks $(p<0.05)$. In addition, in the MOT task, PD-MCI vs. no-PDMCI showed increased stance phase and reduced swing and single-support phases ( $p<0.05$ ), whereas, in the COG task, they exhibited increased stance phase mainly due to longer double-support stance phase duration, increased swing and step variabilities, and reduced velocity $(p>0.05)$.

TABLE 1 | Comparison of demographic and clinical features between patients with Parkinson's disease (PD) without mild cognitive impairment (MCl) (no-PD-MCl) and with $\mathrm{MCl}(\mathrm{PD}-\mathrm{MCl})$.

\begin{tabular}{lccc}
\hline Variables & $\begin{array}{c}\text { No-PD-MCI } \\
(\mathbf{N}=\mathbf{3 5})\end{array}$ & $\begin{array}{c}\text { PD-MCI } \\
(\mathbf{N}=\mathbf{3 2})\end{array}$ & $\boldsymbol{p}$-value \\
\hline Age (years) & $61.70 \pm 7.36$ & $65.70 \pm 9.11$ & 0.053 \\
BMI & $27.30 \pm 2.95$ & $28.2 \pm 3.97$ & 0.286 \\
Disease duration (years) & $4.46 \pm 2.52$ & $5.25 \pm 2.50$ & 0.207 \\
Hoehn and Yahr & $1.75 \pm 0.41$ & $1.92 \pm 0.31$ & 0.068 \\
LEDD (mg) & $495.00 \pm 381.30$ & $563.50 \pm 380.20$ & 0.465 \\
MDS-UPDRS-Part I & $6.54 \pm 4.07$ & $9.03 \pm 7.07$ & 0.088 \\
MDS-UPDRS-Part II & $6.97 \pm 4.68$ & $7.72 \pm 5.64$ & 0.556 \\
MDS-UPDRS-Part III & $20.1 \pm 6.66$ & $24.80 \pm 8.65$ & $\mathbf{0 . 0 1 4}$ \\
MDS-UPDRS-Part IV & $1.86 \pm 3.02$ & $1.44 \pm 2.86$ & 0.420 \\
Total MDS-UPDRS & $35.50 \pm 12.10$ & $43.00 \pm 18.50$ & 0.050
\end{tabular}

PD, Parkinson's Disease; MCl, Mild Cognitive Impairment; BMI, Body Mass Index; LEDD, Levodopa Equivalent Daily Dose; MDS-UPDRS, Movement Disorder Society Unified Parkinson's Disease Rating Scale.

Significant $p$-values are provided in bold. 
TABLE 2 | Comparison of all gait parameters (mean $\pm S D$ ) between patients with PD without $\mathrm{MCl}$ (no-PD-MCl) and with MCl (PD-MCl).

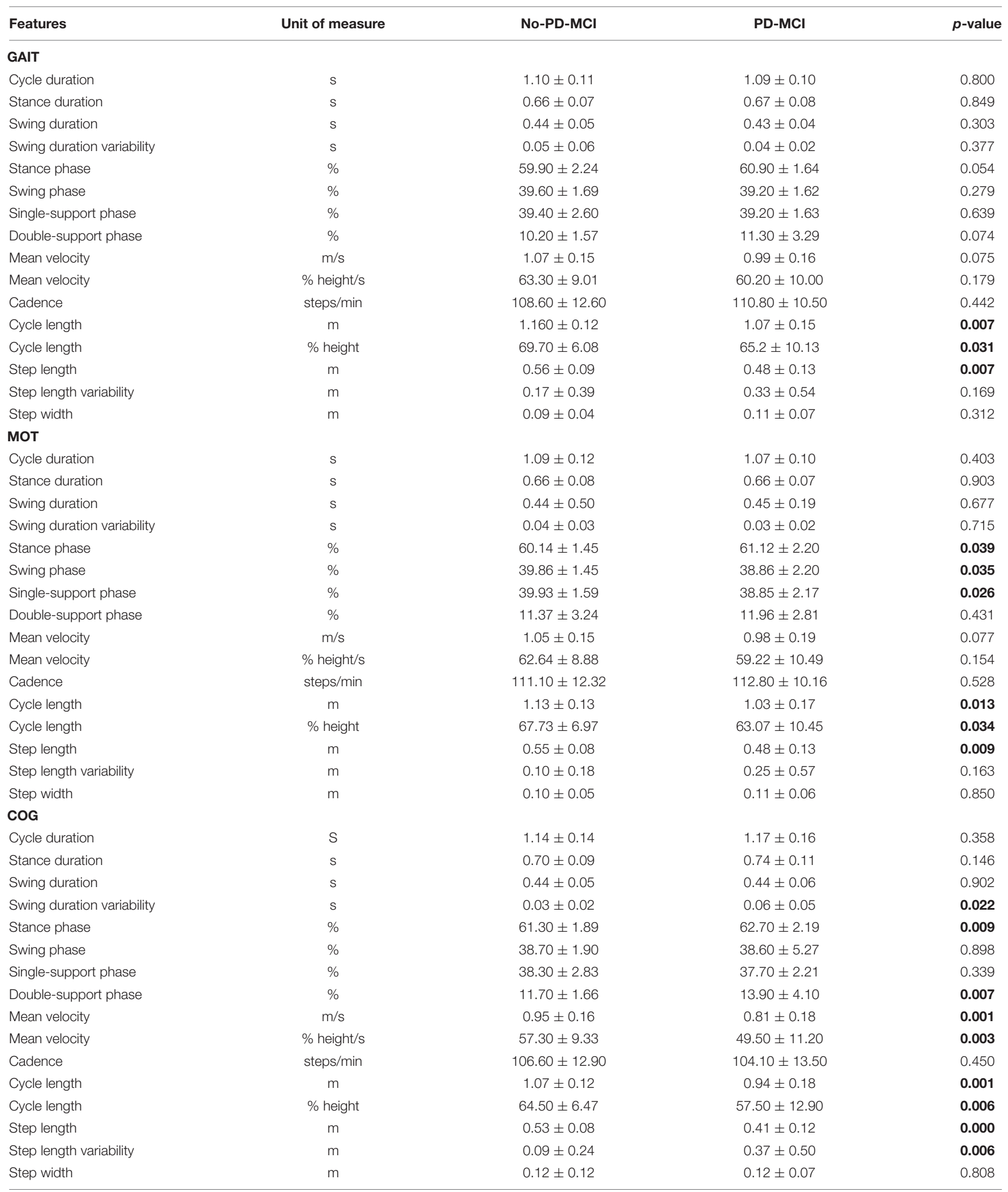

PD, Parkinson's Disease; MCI, Mild Cognitive Impairment; GAIT, normal gait; MOT, walking while carrying a tray with two glasses filled of water; COG, walking while serial subtracting 7s starting from 100.

Significant $p$-values are provided in bold. 


\section{No-Parkinson's Disease-Mild Cognitive Impairment vs. Single-Domain Parkinson's Disease-Mild Cognitive Impairment vs. Multiple-Domain Parkinson's Disease-Mild Cognitive Impairment}

When subtyping PD-MCI according to the number of affected cognitive domains, 7 patients were categorized as singledomain PD-MCI and 25 patients as multiple-domain PD-MCI. Table 3 shows the comparison of all spatial and temporal gait parameters among no-PD-MCI, single-domain PD-MCI, and multiple-domain PD-MCI with subsequent post-hoc tests when appropriate.

In all three tasks, step and cycle lengths showed statistically significant differences among the three groups $(p<0.05)$; post hoc comparisons revealed significantly shortened step and cycle lengths in multiple-domain PD-MCI vs. no-PD-MCI $(p<0.05)$. Moreover, in the MOT task, the stance phase, the swing phase, the single-support phase, and velocity resulted statistically different among the three groups; the post hoc analysis disclosed reduced swing and single-support phases in multiple-domain PD-MCI vs. no-PD-MCI $(p<0.05)$.

In the COG task, swing phase and step length variabilities, the stance and swing phases, the double-support phase, and velocity were significantly different among the three groups $(p<0.05)$; the post hoc analysis revealed increased swing phase and step length variabilities, longer stance and double-support phases, and reduced velocity in multiple-domain PD-MCI as compared to no-PD-MCI $(p<0.05)$.

\section{No-Parkinson's Disease-Mild Cognitive Impairment vs. Amnestic Parkinson's Disease-Mild Cognitive Impairment vs. Non-amnestic Parkinson's Disease-Mild Cognitive Impairment}

When subtyping PD-MCI based on the presence of memory impairment, 21 patients were diagnosed as amnestic PD-MCI and 11 patients as non-amnestic PD-MCI. Table 4 shows the comparison of all spatial and temporal gait parameters among no-PD-MCI, amnestic PD-MCI, and non-amnestic PD-MCI using subsequent post-hoc tests when appropriate.

In all three tasks, the cycle length normalized for height resulted to be significantly different among the three groups $(p<0.05)$; in the GAIT and COG tasks, post hoc comparisons revealed significantly shortened cycle length normalized for height in amnestic PD-MCI vs. no-PD-MCI $(p<0.05)$. In addition, in the COG task, swing phase and step length variabilities, stance duration, stance and double-support phases, velocity, and cycle and step lengths were significantly different among the three groups $(p<0.05)$. The post hoc analysis revealed increased swing phase and step length variabilities, increased stance and double-support phases, reduced velocity, and shortened cycle and step lengths in amnestic PD-MCI as compared to no-PD-MCI $(p<0.05)$; the post hoc analysis also unveiled shortened step length in non-amnestic PD-MCI vs. no-PD-MCI $(p<0.001)$.

\section{Exploratory Correlation Analysis Between Gait Parameters and Neuropsychological Test Scores}

The correlation analysis was performed between the neuropsychological tests scores and the spatiotemporal gait parameters under the COG dual task, as these gait variables were most related to cognition in the previous analysis. Significant correlations were obtained for the global cognition scale MoCA, which correlated with the cycle duration $(r=-0.664, p=0.004)$, the stance duration $(r=-0.678, p=0.003)$, the stance phase $(r=-0.540, p=0.025)$, the swing phase $(r=0.554, p=0.021)$, the single-support phase $(r=0.554, p=0.021)$, the double-support phase $(r=-0.537, p=0.026)$, and the cadence $(r=-0.660$, $p=0.004)$.

Among the domain-specific neuropsychological tests, only the Benton's Judgment of Line Orientation Test exhibited a significant correlation (between 0.500 and 0.600 ) with a gait parameter, namely mean velocity $(r=0.550, p<0.001)$ (Supplementary Figure 1).

\section{DISCUSSION}

To our knowledge, this is the first study evaluating quantitative walking parameters in MCI subtypes in PD. After confirming that PD-MCI is associated with the dysfunction of several spatial and temporal gait parameters, especially in dual-task conditions, in this study, we showed that multiple-domain PD-MCI and amnestic PD-MCI are coupled with worse gait patterns.

\section{Comparison of Gait Features in Parkinson's Disease-Mild Cognitive Impairment vs. No-Parkinson's Disease-Mild Cognitive Impairment}

Consistent with previous findings (Amboni et al., 2012, 2018; Morris et al., 2016, 2019), patients with PD-MCI as compared to patients with no-PD-MCI showed predominant dysfunctions on spatial gait features, i.e., reduced cycle and step length, which were evident in both single and dual task. Under dual-task conditions, PD-MCI exhibited increased stance phase, mainly in double support, and augmented the measures of variability, i.e., swing duration variability and step length variability, which are all indicators of dynamic instability (Plotnik et al., 2011). These findings further support the detrimental effect of the dual task on gait performance, especially on dynamic balance, in patients with cognitive decline (Amboni et al., 2013; Bishnoi and Hernandez, 2020; Ricciardi et al., 2020) with a subsequent increased risk of falling. In fact, the sensitizing role of dual task relies on its peculiar mechanism that leads to a competition for attention resources that collapse when a cognitive reserve is reduced (Amboni et al., 2013). It is worth noting that, since all patients were on medication during gait analysis, the observed gait variable dysfunctions may mirror their levodopa-resistant nature. 
TABLE 3 | Comparison of all gait parameters (mean \pm SD) among no-PD-MCl, single-domain PD-MCl, and multiple-domain PD-MCl.

\begin{tabular}{|c|c|c|c|c|c|}
\hline Features & $\begin{array}{c}\text { No-PD-MCl } \\
\text { (0) }\end{array}$ & $\begin{array}{c}\text { Single-domain } \\
\text { PD-MCI } \\
\text { (1) }\end{array}$ & $\begin{array}{c}\text { Multiple-domain } \\
\text { PD-MCI } \\
\text { (2) }\end{array}$ & $\begin{array}{c}p \text {-value } \\
\text { Kruskal-wallis }\end{array}$ & $\begin{array}{l}p \text {-value } \\
\text { post hoc }\end{array}$ \\
\hline \multicolumn{6}{|l|}{ GAIT } \\
\hline Cycle duration & $1.10 \pm 0.11$ & $1.09 \pm 0.10$ & $1.09 \pm 0.10$ & 0.986 & \\
\hline Stance duration & $0.66 \pm 0.07$ & $0.66 \pm 0.06$ & $0.67 \pm 0.08$ & 0.998 & \\
\hline Swing duration & $0.44 \pm 0.05$ & $0.44 \pm 0.04$ & $0.43 \pm 0.03$ & 0.692 & \\
\hline Swing duration variability & $0.05 \pm 0.06$ & $0.05 \pm 0.01$ & $0.03 \pm 0.02$ & 0.101 & \\
\hline Stance phase & $59.90 \pm 2.24$ & $60.10 \pm 1.07$ & $61.00 \pm 1.70$ & 0.159 & \\
\hline Swing phase & $39.60 \pm 1.69$ & $39.90 \pm 1.05$ & $39.00 \pm 1.70$ & 0.337 & \\
\hline Single-support phase & $39.40 \pm 2.60$ & $39.90 \pm 1.05$ & $39.00 \pm 1.70$ & 0.311 & \\
\hline Double-support phase & $10.20 \pm 1.57$ & $10.20 \pm 1.47$ & $11.5 \pm 3.56$ & 0.279 & \\
\hline Mean velocity & $1.07 \pm 0.15$ & $1.08 \pm 0.08$ & $0.98 \pm 0.17$ & 0.142 & \\
\hline Mean velocity\% $\mathrm{h} / \mathrm{s}$ & $63.30 \pm 9.01$ & $64.00 \pm 5.05$ & $59.30 \pm 10.7$ & 0.266 & \\
\hline Cadence & $108.60 \pm 12.60$ & $110.80 \pm 9.67$ & $110.80 \pm 10.90$ & 0.829 & \\
\hline Cycle length & $1.16 \pm 0.12$ & $1.18 \pm 0.04$ & $1.05 \pm 0.15$ & 0.020 & $2-00.032$ \\
\hline Cycle length \% h & $69.70 \pm 6.08$ & $69.30 \pm 1.73$ & $64.20 \pm 11.00$ & 0.005 & $2-00.005$ \\
\hline Step length & $0.56 \pm 0.09$ & $0.57 \pm 0.07$ & $0.46 \pm 0.13$ & 0.012 & $2-00.015$ \\
\hline Step length variability & $0.17 \pm 0.39$ & $0.13 \pm 0.22$ & $0.38 \pm 0.59$ & 0.263 & \\
\hline Step width & $0.09 \pm 0.04$ & $0.10 \pm 0.05$ & $0.11 \pm 0.07$ & 0.933 & \\
\hline \multicolumn{6}{|l|}{ МOT } \\
\hline Cycle duration & $1.09 \pm 0.12$ & $1.05 \pm 0.09$ & $1.07 \pm 0.10$ & 0.632 & \\
\hline Stance duration & $0.66 \pm 0.08$ & $0.63 \pm 0.06$ & $0.66 \pm 0.07$ & 0.478 & \\
\hline Swing duration & $0.44 \pm 0.50$ & $0.42 \pm 0.04$ & $0.46 \pm 0.21$ & 0.391 & \\
\hline Swing duration variability & $0.04 \pm 0.03$ & $0.03 \pm 0.03$ & $0.03 \pm 0.02$ & 0.381 & \\
\hline Stance phase & $60.10 \pm 1.45$ & $59.50 \pm 2.17$ & $61.50 \pm 2.07$ & 0.024 & $1-20.0960-20.064$ \\
\hline Swing phase & $39.90 \pm 1.45$ & $40.50 \pm 2.17$ & $38.50 \pm 2.07$ & 0.019 & $2-00.050$ \\
\hline Single-support phase & $39.90 \pm 1.59$ & $40.50 \pm 2.19$ & $38.50 \pm 2.02$ & 0.015 & 2-0 0.039 \\
\hline Double-support phase & $11.40 \pm 3.24$ & $11.40 \pm 3.39$ & $12.10 \pm 2.72$ & 0.210 & \\
\hline Mean velocity & $1.05 \pm 0.15$ & $1.12 \pm 0.17$ & $0.94 \pm 0.19$ & 0.034 & 2-0 0.088 2-10.119 \\
\hline Mean velocity $\% \mathrm{~h} / \mathrm{s}$ & $62.60 \pm 8.90$ & $66.50 \pm 9.50$ & $57.60 \pm 10.15$ & 0.034 & $2-00.105$ 2-1 0.101 \\
\hline Cadence & $111.10 \pm 12.30$ & $115.33 \pm 9.90$ & $112.30 \pm 10.30$ & 0.665 & \\
\hline Cycle length & $1.13 \pm 0.13$ & $1.16 \pm 0.08$ & $1.00 \pm 0.17$ & 0.008 & $2-00.015$ \\
\hline Cycle length \% h & $67.70 \pm 6.90$ & $68.80 \pm 5.70$ & $61.70 \pm 10.90$ & 0.003 & $2-00.003$ \\
\hline Step length & $0.55 \pm 0.08$ & $0.56 \pm 0.06$ & $0.46 \pm 0.13$ & 0.018 & $2-00.015$ \\
\hline Step length variability & $0.10 \pm 0.18$ & $0.13 \pm 0.22$ & $0.28 \pm 0.62$ & 0.659 & \\
\hline Step width & $0.10 \pm 0.05$ & $0.11 \pm 0.06$ & $0.11 \pm 0.06$ & 0.815 & \\
\hline \multicolumn{6}{|l|}{ COG } \\
\hline Cycle duration & $1.14 \pm 0.14$ & $1.18 \pm 0.16$ & $1.18 \pm 0.16$ & 0.677 & \\
\hline Stance duration & $0.70 \pm 0.09$ & $0.72 \pm 0.08$ & $0.74 \pm 0.11$ & 0.396 & \\
\hline Swing duration & $0.44 \pm 0.05$ & $0.46 \pm 0.09$ & $0.43 \pm 0.05$ & 0.998 & \\
\hline Swing duration variability & $0.03 \pm 0.02$ & $0.05 \pm 0.04$ & $0.06 \pm 0.05$ & 0.024 & $2-00.022$ \\
\hline Stance phase & $61.30 \pm 1.89$ & $61.60 \pm 2.21$ & $62.90 \pm 2.15$ & 0.019 & $2-00.015$ \\
\hline Swing phase & $38.70 \pm 1.90$ & $43.60 \pm 10.70$ & $37.40 \pm 2.02$ & 0.016 & $2-00.0612-10.052$ \\
\hline Single-support phase & $38.30 \pm 2.83$ & $38.70 \pm 2.41$ & $37.50 \pm 2.14$ & 0.137 & \\
\hline Double-support phase & $11.70 \pm 1.66$ & $13.90 \pm 3.65$ & $13.90 \pm 4.26$ & 0.030 & 2-0 0.039 \\
\hline Mean velocity & $0.95 \pm 0.16$ & $0.92 \pm 0.12$ & $0.79 \pm 0.19$ & 0.002 & $2-00.001$ \\
\hline Mean velocity $\% \mathrm{~h} / \mathrm{s}$ & $57.30 \pm 9.33$ & $53.80 \pm 7.57$ & $48.50 \pm 11.80$ & 0.008 & $2-00.006$ \\
\hline Cadence & $106.50 \pm 12.90$ & $104.20 \pm 12.90$ & $104.10 \pm 13.90$ & 0.732 & \\
\hline Cycle length & $1.07 \pm 0.12$ & $1.05 \pm 0.09$ & $0.92 \pm 0.19$ & 0.002 & $2-00.002$ \\
\hline Cycle length \% h & $64.50 \pm 6.47$ & $62.1 \pm 7.28$ & $56.40 \pm 13.80$ & 0.001 & $2-00.001$ \\
\hline Step length & $0.53 \pm 0.08$ & $0.44 \pm 0.10$ & $0.40 \pm 0.12$ & 0.000 & $2-00.000$ \\
\hline Step length variability & $0.09 \pm 0.24$ & $0.39 \pm 0.39$ & $0.37 \pm 0.53$ & 0.002 & $2-00.004$ \\
\hline Step width & $0.12 \pm 0.12$ & $0.14 \pm 0.11$ & $0.11 \pm 0.07$ & 0.796 & \\
\hline
\end{tabular}

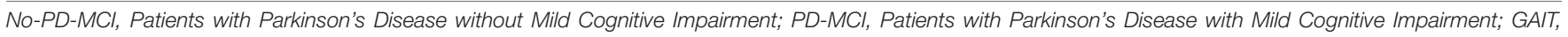
normal gait; MOT, walking while carrying a tray with two glasses filled of water; COG, walking while serial subtracting 7 s starting from 100.

Significant $p$-values are provided in bold. 
TABLE 4 | Comparison of all gait parameters (mean $\pm S D$ ) among no-PD-MCl, amnestic PD-MCl, and non-amnestic PD-MCl.

\begin{tabular}{|c|c|c|c|c|c|}
\hline Features & $\begin{array}{c}\text { No-PD-MCI } \\
(0)\end{array}$ & $\begin{array}{l}\text { Amnestic } \\
\text { PD-MCI } \\
\text { (1) }\end{array}$ & $\begin{array}{c}\text { Non-amnestic } \\
\text { PD-MCI } \\
\text { (2) }\end{array}$ & $\begin{array}{c}p \text {-value } \\
\text { Kruskal-wallis }\end{array}$ & $\begin{array}{l}\text { p-value } \\
\text { post hoc }\end{array}$ \\
\hline \multicolumn{6}{|l|}{ GAIT } \\
\hline Cycle duration & $1.10 \pm 0.11$ & $1.11 \pm 0.10$ & $1.05 \pm 0.10$ & 0.272 & \\
\hline Stance duration & $0.66 \pm 0.07$ & $0.68 \pm 0.08$ & $0.64 \pm 0.06$ & 0.235 & \\
\hline Swing duration & $0.44 \pm 0.05$ & $0.43 \pm 0.03$ & $0.42 \pm 0.05$ & 0.337 & \\
\hline Swing duration variability & $0.05 \pm 0.06$ & $0.04 \pm 0.02$ & $0.03 \pm 0.02$ & 0.995 & \\
\hline Stance phase & $59.90 \pm 2.24$ & $61.1 \pm 1.75$ & $60.40 \pm 1.33$ & 0.147 & \\
\hline Swing phase & $39.60 \pm 1.69$ & $38.90 \pm 1.75$ & $39.60 \pm 1.32$ & 0.324 & \\
\hline Single-support phase & $39.40 \pm 2.60$ & $38.90 \pm 1.76$ & $39.70 \pm 1.31$ & 0.304 & \\
\hline Double-support phase & $10.20 \pm 1.57$ & $11.80 \pm 3.83$ & $10.20 \pm 1.53$ & 0.121 & \\
\hline Mean velocity & $1.07 \pm 0.15$ & $0.97 \pm 0.18$ & $1.04 \pm 0.10$ & 0.333 & \\
\hline Mean velocity $\% \mathrm{~h} / \mathrm{s}$ & $63.3 \pm 9.01$ & $59.10 \pm 11.70$ & $62.20 \pm 5.41$ & 0.368 & \\
\hline Cadence & $108.60 \pm 12.60$ & $108.50 \pm 10.10$ & $115.00 \pm 10.60$ & 0.241 & \\
\hline Cycle length & $1.16 \pm 0.12$ & $1.07 \pm 0.17$ & $1.09 \pm 0.12$ & 0.125 & \\
\hline Cycle length \% h & $69.7 \pm 6.08$ & $65.10 \pm 11.70$ & $65.40 \pm 6.68$ & 0.022 & $1-00.034$ \\
\hline Step length & $0.56 \pm 0.10$ & $0.48 \pm 0.13$ & $0.47 \pm 0.14$ & 0.065 & \\
\hline Step length variability & $0.17 \pm 0.39$ & $0.34 \pm 0.61$ & $0.32 \pm 0.42$ & 0.254 & \\
\hline Step width & $0.09 \pm 0.04$ & $0.09 \pm 0.05$ & $0.14 \pm 0.09$ & 0.508 & \\
\hline \multicolumn{6}{|l|}{ MOT } \\
\hline Cycle duration & $1.09 \pm 0.12$ & $1.09 \pm 0.09$ & $1.03 \pm 0.10$ & 0.220 & \\
\hline Stance duration & $0.66 \pm 0.08$ & $0.68 \pm 0.08$ & $0.62 \pm 0.05$ & 0.116 & \\
\hline Swing duration & $0.44 \pm 0.50$ & $0.47 \pm 0.23$ & $0.41 \pm 0.05$ & 0.204 & \\
\hline Swing duration variability & $0.04 \pm 0.03$ & $0.03 \pm 0.02$ & $0.03 \pm 0.02$ & 0.928 & \\
\hline Stance phase & $60.10 \pm 1.45$ & $61.50 \pm 2.31$ & $60.50 \pm 1.91$ & 0.174 & \\
\hline Swing phase & $39.90 \pm 1.45$ & $38.50 \pm 2.30$ & $39.50 \pm 1.91$ & 0.157 & \\
\hline Single-support phase & $39.90 \pm 1.59$ & $28.50 \pm 2.28$ & $39.50 \pm 1.88$ & 0.143 & \\
\hline Double-support phase & $11.40 \pm 3.24$ & $12.20 \pm 3.06$ & $11.60 \pm 2.35$ & 0.387 & \\
\hline Mean velocity & $1.05 \pm 0.15$ & $0.95 \pm 0.22$ & $1.01 \pm 0.12$ & 0.245 & \\
\hline Mean velocity $\% \mathrm{~h} / \mathrm{s}$ & $62.60 \pm 8.90$ & $58.10 \pm 10.30$ & $61.3 \pm 6.59$ & 0.311 & \\
\hline Cadence & $111.10 \pm 12.30$ & $110.40 \pm 9.00$ & $117.60 \pm 10.90$ & 0.198 & \\
\hline Cycle length & $1.13 \pm 0.13$ & $1.03 \pm 0.19$ & $1.05 \pm 0.12$ & 0.086 & \\
\hline Cycle length \% h & $67.70 \pm 6.97$ & $63.20 \pm 12.10$ & $62.90 \pm 6.96$ & 0.022 & 1-0 0.076 2-0 0.076 \\
\hline Step length & $0.55 \pm 0.08$ & $0.48 \pm 0.13$ & $0.48 \pm 0.12$ & 0.072 & \\
\hline Step length variability & $0.10 \pm 0.18$ & $0.27 \pm 0.66$ & $0.22 \pm 0.34$ & 0.637 & \\
\hline Step width & $0.10 \pm 0.05$ & $0.10 \pm 0.06$ & $0.11 \pm 0.06$ & 0.839 & \\
\hline \multicolumn{6}{|l|}{ COG } \\
\hline Cycle duration & $1.14 \pm 0.14$ & $1.21 \pm 0.15$ & $1.11 \pm 0.16$ & 0.108 & \\
\hline Stance duration & $0.70 \pm 0.09$ & $0.77 \pm 0.10$ & $0.69 \pm 0.10$ & 0.048 & $1-00.095$ \\
\hline Swing duration & $0.44 \pm 0.05$ & $0.45 \pm 0.05$ & $0.43 \pm 0.07$ & 0.356 & \\
\hline Swing duration variability & $0.03 \pm 0.02$ & $0.06 \pm 0.06$ & $0.04 \pm 0.02$ & 0.021 & $1-00.021$ \\
\hline Stance phase & $61.30 \pm 1.90$ & $63.20 \pm 1.9$ & $61.80 \pm 2.40$ & 0.013 & $1-00.009$ \\
\hline Swing phase & $38.70 \pm 1.90$ & $38.50 \pm 6.40$ & $38.60 \pm 1.90$ & 0.129 & \\
\hline Single-support phase & $38.30 \pm 2.80$ & $37.30 \pm 2.20$ & $38.60 \pm 1.90$ & 0.095 & \\
\hline Double-support phase & $11.70 \pm 1.70$ & $14.1 \pm 4.20$ & $13.70 \pm 4.00$ & 0.024 & $1-00.025$ \\
\hline Mean velocity & $0.95 \pm 0.16$ & $0.79 \pm 0.19$ & $0.84 \pm 0.17$ & 0.005 & $1-00.006$ \\
\hline Mean velocity $\% \mathrm{~h} / \mathrm{s}$ & $57.30 \pm 9.30$ & $48.70 \pm 11.70$ & $51.00 \pm 10.50$ & 0.011 & $1-00.011$ \\
\hline Cadence & $106.50 \pm 12.90$ & $100.90 \pm 12.10$ & $110.00 \pm 14.60$ & 0.127 & \\
\hline Cycle length & $1.07 \pm 0.12$ & $0.94 \pm 0.17$ & $0.94 \pm 0.22$ & 0.009 & $1-00.015$ \\
\hline Cycle length \% h & $64.50 \pm 6.50$ & $57.90 \pm 13.10$ & $56.70 \pm 13.40$ & 0.004 & $1-00.006$ \\
\hline Step length & $0.53 \pm 0.08$ & $0.40 \pm 0.13$ & $0.42 \pm 0.10$ & 0.000 & $2-00.0091-00.001$ \\
\hline Step length variability & $0.09 \pm 0.24$ & $0.44 \pm 0.57$ & $0.23 \pm 0.32$ & 0.001 & $1-00.001$ \\
\hline Step width & $0.11 \pm 0.12$ & $0.11 \pm 0.07$ & $0.12 \pm 0.08$ & 0.800 & \\
\hline
\end{tabular}

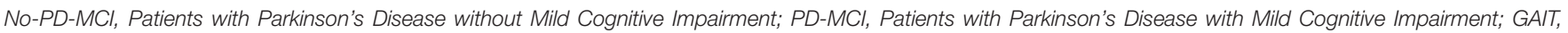
normal gait; MOT, walking while carrying a tray with two glasses filled of water; COG, walking while serial subtracting 7 s starting from 100.

Significant $p$-values are provided in bold. 


\section{Comparison of Gait Features in Parkinson's Disease-Mild Cognitive Impairment Subtypes}

While comparing PD-MCI subtypes based on the number of affected cognitive domains, the magnitude of gait impairment resulted to be directly related to the severity of cognitive dysfunction. In particular, multiple-domain PD-MCI vs. no-PDMCI showed impaired spatial gait measures, such as shortened step and cycle length, in all three tasks, and increased measures of dynamic unbalance, similar to raised variability measures and increased double-support phase, during dual tasks. On the contrary, single-domain PD-MCI as compared to no-PD-MCI did not display any significant difference on gait features neither in single nor in dual task. Nevertheless, the post hoc analysis did not reveal even any significant difference when comparing gait patterns in patients with single-domain PD-MCI and patients with multiple-domain PD-MCI, thus suggesting a spectrum of dysfunction concurrently involving gait and cognition spanning across cognitively intact patients with $\mathrm{PD}$, patients with singledomain PD, and patients with multiple-domain PD.

When confronting PD-MCI subtypes based on the nature of the affected cognitive domain, such as the presence of memory dysfunction, patients with amnestic PD-MCI, as compared to patients with no-PD-MCI and patients with non-amnestic PD-MCI, mainly showed increased dynamic instability during the COG task, evidenced by augmented variability measures and increased double-support phase. In addition, patients with both amnestic PD-MCI and non-amnestic PD-MCI vs. patients with no-PD-MCI subjects displayed reduced step length during the COG task. Again, we might speculate on the pairwise comparisons and infer a grading of gait dysfunction that is the greatest in patients with amnestic PD-MCI, intermediate in patients with non-amnestic PD-MCI, and the smallest in patients with no-PD-MCI. The present findings would be consistent with the progression of neurodegeneration from the anterior to the posterior cortical areas, likely involving also non-dopaminergic networks (Devos et al., 2010), which expresses with the concurrent occurrence of peculiar gait and neuropsychological dysfunction (Figure 1).

As regards walking abnormalities, previous imaging studies (Beauchet et al., 2014; Lo et al., 2017) showed that dynamic steadiness is linked to posterior brain networks, whereas more recent findings suggested the involvement of widespread cortical areas important for sensory, cognitive, and motor functions (Jayakody et al., 2020). In fact, dynamic stability is a complex activity requiring integration and control of multiple components, such as the integration of different sensory information, postural adjustments, and motor planning. As concerns cognitive dysfunction, several studies in patients with PD have demonstrated that, as the cognitive decline progresses, the cortical volume, especially in temporoparietal regions, decreases with parallel decay on memory (Lee et al., 2010; Mak et al., 2015; Pereira et al., 2015; Yildiz et al., 2015). In addition, since both multiple-domain PD-MCI and amnestic PD-MCI have been associated with the increased risk of conversion to dementia (Janvin et al., 2006; Hoogland et al., 2017;

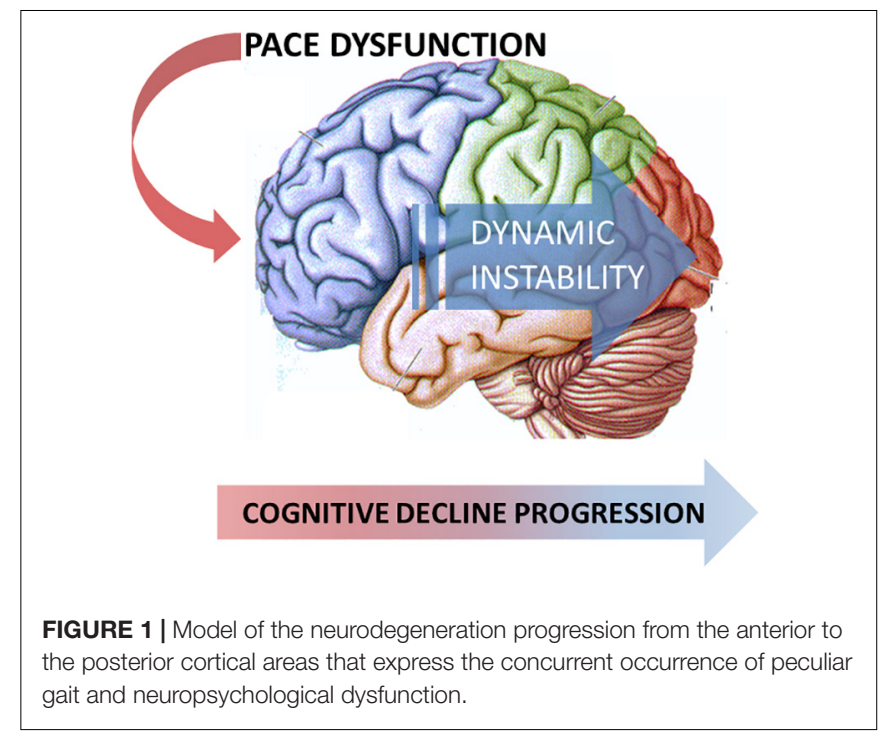

Chung et al., 2019), the increased dynamic instability, especially in dual task, might represent a possible marker of progression toward PDD. Nevertheless, further longitudinal studies are needed to investigate this speculation.

Due to the cross-sectional design of this study, these findings cannot allow any assumption about a causative relationship between posterior cortical-based cognitive deficit and dynamic instability. In other words, our findings show an association between distinctive neuropsychological profiles and specific walking abnormalities, but they cannot establish whether peculiar cognitive dysfunctions can directly contribute to the expression of particular gait patterns.

As regards the exploratory correlation analysis between spatiotemporal gait parameters during the COG dual-task and neuropsychological tests scores, only the MoCA score, a global cognition rating, correlated with gait parameters mirroring dynamic instability, thus further supporting the relationship between the severity of cognitive dysfunction and the extent of gait impairment. In fact, when analyzing correlations between domain-specific neuropsychological tests and gait variables, we found only a correlation between a visuospatial test, namely, the Benton's Judgment of Line Orientation Test, and velocity, a raw measure underlain by multiple gait adaptations. The latter findings are not surprising since the relationship between gait and cognition would reflect common network dysfunction, expressing a collapse on both gait and cognitive skills, probably without strict correlations between walking variables and isolated cognitive test scores. Further studies on larger samples are needed to better explore this issue.

This study has some limitations. First, due to the relatively small sample size, we could not classify and, therefore, compare PD-MCI subtypes immediately according to the four categories, namely, single amnesic PD-MCI, single non-amnesic PD-MCI, multiple amnesic PD-MCI, and multiple non-amnesic PD. Although we recognized that this classification could directly show distinctive gait patterns in PD-MCI subtypes, we envisaged that the separate comparisons, i.e., single- vs. multiple-domain 
PD-MCI and amnestic vs. non-amnestic PD-MCI, may represent a reliable method to capture the relative burden of the quantity and quality of cognitive dysfunction on walking features. Second, since the single-domain PD-MCI group included only seven patients, this could have introduced a bias in the interpretation of data; however, this low prevalence is fairly consistent with the findings of a recent meta-analysis reporting that single-domain subtype occurs only in about one-third of patients with PD-MCI (Baiano et al., 2020). Third, we did not include an age-matched control group in our analysis. This arguably could better highlight the difference between the PD-MCI subtypes; nevertheless, a direct comparison with a control group was beyond the purpose of this study. Finally, when comparing demographic and clinical features, we found that PD-MCI vs. no-PD-MCI showed a significant difference on MDS-UPDRS III and a trend toward significance on age, and this might have had an influence on our findings. Nevertheless, such differences reflect two of the main clinical features associated with MCI, namely, older age and more severe motor symptoms, as consistently reported in the literature (Baiano et al., 2020), suggesting a tight relationship among MCI, age, and more severe motor impairment. In other terms, the gait patterns observed in MCI and its subtypes reflect the contribution of all these features that are indissociable in a real-life clinical setting.

\section{CONCLUSION}

In conclusion, this study demonstrates that PD-MCI subtypes are associated with different gait patterns. Notably, both the magnitude of cognitive impairment and the presence of memory dysfunction are associated with the increased measures of dynamic unbalance, especially in dual-task condition, likely mirroring the progressive involvement of posterior cortical networks that are concurrently revealed by distinctive cognitive profiles and walking patterns. Additionally, our findings may suggest speculative clues for integrated therapeutic approaches, which could range from cognitive therapy (cognitive training

\section{REFERENCES}

Aarsland, D., Creese, B., Politis, M., Chaudhuri, K. R., Ffytche, D. H., Weintraub, D., et al. (2017). Cognitive decline in Parkinson disease. Nat. Rev. Neurol. 13, $217-231$.

Amboni, M., Barone, P., and Hausdorff, J. M. (2013). Cognitive contributions to gait and falls: evidence and implications. Mov. Disord 28, 1520-1533. doi: $10.1002 / \mathrm{mds} .25674$

Amboni, M., Barone, P., Iuppariello, L., Lista, I., Tranfaglia, R., Fasano, A., et al. (2012). Gait patterns in Parkinsonian patients with or without mild cognitive impairment. Mov. Disord 27, 1536-1543. doi: 10.1002/mds. 25165

Amboni, M., Iuppariello, L., Iavarone, A., Fasano, A., Palladino, R., Rucco, R., et al. (2018). Step length predicts executive dysfunction in Parkinson's disease: a 3year prospective study. J. Neurol. 265, 2211-2220. doi: 10.1007/s00415-0188973-x

Baiano, C., Barone, P., Trojano, L., and Santangelo, G. (2020). Prevalence and clinical aspects of mild cognitive impairment in Parkinson's disease: a metaanalysis. Mov. Disord 35, 45-54. doi: 10.1002/mds.27902 interventions and use of cognitive pharmacological therapy) for improving walking performance to specific walking programs for enhancing cognitive function.

\section{DATA AVAILABILITY STATEMENT}

The raw data supporting the conclusions of this article will be made available by the authors, without undue reservation.

\section{ETHICS STATEMENT}

The studies involving human participants were reviewed and approved by Campania Sud, the Reference Ethics Committee of the Center for Neurodegenerative Diseases of the University of Salerno. The patients/participants provided their written informed consent to participate in this study.

\section{AUTHOR CONTRIBUTIONS}

MA and PB contributed to conception and design of the study. $\mathrm{CR}$, SC, AV, and GR organized the database. CR, LD, and MC performed the statistical analysis. MA and CR wrote the first draft of the manuscript. MP and GS wrote sections of the manuscript. All authors contributed to manuscript revision, read, and approved the submitted version.

\section{SUPPLEMENTARY MATERIAL}

The Supplementary Material for this article can be found online at: https://www.frontiersin.org/articles/10.3389/fnagi. 2022.781480/full\#supplementary-material

Supplementary Figure 1 | Scatterplot representing the correlation between the Benton's Judgment of Line Orientation Test score and mean velocity under COG dual task.
Barbarotto, R., Laiacona, M., Frosio, R., Vecchio, M., Farinato, A., and Capitani, E. (1998). A normative study on visual reaction times and two Stroop colour-word tests. Ital. J. Neurol. Sci. 19, 161-170. doi: 10.1007/BF00831566

Barone, P., Aarsland, D., Burn, D., Emre, M., Kulisevsky, J., and Weintraub, D. (2011). Cognitive impairment in nondemented Parkinson's disease. Mov. Dis. 26, 2483-2495.

Beauchet, O., Annweiler, C., Celle, S., Bartha, R., Barthélémy, J. C., and Roche, F. (2014). Higher gait variability is associated with decreased parietal gray matter volume among healthy older adults. Brain Topogr. 27, 293-295. doi: 10.1007/ s10548-013-0293-y

Bishnoi, A., and Hernandez, M. E. (2020). Dual task walking costs in older adults with mild cognitive impairment: a systematic review and meta-analysis. Aging Ment. Health 6, 1-12. doi: 10.1080/13607863.2020.1802576

Caffarra, P., Vezzadini, G., Dieci, F., Zonato, F., and Venneri, A. (2002). ReyOsterrieth complex figure: normative values in an Italian population sample. Neurol. Sci. 22, 443-447. doi: 10.1007/s100720200003

Caltagirone, C., Gainotti, G., Masullo, C., and Miceli, G. (1979). Validity of some neuropsychological test in the assessment of mental deterioration. Acta Psychiatr. Scand. 60, 50-56. 
Capasso, R., and Miceli, G. (2001). Esame Neuropsicologico per l'Afasia, ENPA. Milan: Springer.

Chen, P. H., Lien, C. W., Wu, W. C., Lee, L. S., and Shaw, J. S. (2020). GaitBased Machine Learning for Classifying Patients with Different Types of Mild Cognitive Impairment. J. Med. Syst. 44:107. doi: 10.1007/s10916-020-01578-7

Chung, S. J., Park, Y. H., Yun, H. J., Kwon, H., Yoo, H. S., Sohn, Y. H., et al. (2019). Clinical relevance of amnestic versus non-amnestic mild cognitive impairment subtyping in Parkinson's disease. Eur. J. Neurol. 26, 766-773. doi: 10.1111/ene. 13886

Davis, R. B. III, Ounpuu, S., Tyburski, D., and Gage, J. R. (1991). A gait analysis data collection and reduction technique. Hum. Mov. Sci. 10, 575-587. doi: 10.1016/0167-9457(91)90046-z

de Oliveira Silva, F., Ferreira, J. V., Plácido, J., Chagas, D., Praxedes, J., Guimarães, C., et al. (2020). Gait analysis with videogrammetry can differentiate healthy elderly, mild cognitive impairment, and Alzheimer's disease: a cross-sectional study. Exp. Geront. 131:110816. doi: 10.1016/j.exger.2019.110816

Devos, D., Defebvre, L., and Bordet, R. (2010). Dopaminergic and nondopaminergic pharmacological hypotheses for gait disorders in Parkinson's disease. Fundam Clin. Pharmacol. 24, 407-421. doi: 10.1111/j.1472-8206.2009. 00798.x

Emre, M., Aarsland, D., Brown, R., Burn, D. J., Duyckaerts, C., Mizuno, Y., et al. (2007). Clinical diagnostic criteria for dementia associated with Parkinson's disease. Mov. Disord. 22, 1689-1707. doi: 10.1002/mds.21507

Ferracuti, S., Sacco, R., Cannoni, E., Hufty, A. M., Silvan, A. B., Hamster, K., et al. (2000). Contributi per un Assessment Neuropsicologico di Benton AL. Florence: Organizzazioni Speciali.

Giovagnoli, A. R., Del Pesce, M., Mascheroni, S., Simoncelli, M., Laiacona, M., and Capitani, E. (1996). Trail making test: normative values from 287 normal adult controls. Ital. J. Neurol. Sci. 17, 305-309. doi: 10.1007/BF01997792

Hoogland, J., Boel, J. A., de Bie, R. M. A., Geskus, R. B., Schmand, B. A., DalrympleAlford, J. C., et al. (2017). Mild cognitive impairment as a risk factor for Parkinson's disease dementia. Mov. Disord 32, 1056-1065. doi: 10.1002/mds. 27002

Janvin, C. C., Larsen, J. P., Aarsland, D., and Hugdahl, K. (2006). Subtypes of mild cognitive impairment in Parkinson's disease: progression to dementia. Mov. Disord. 21, 1343-1349. doi: 10.1002/mds.20974

Jayakody, O., Breslin, M., Beare, R., Blumen, H. M., Srikanth, V. K., and Callisaya, M. L. (2020). Regional Associations of Cortical Thickness With Gait VariabilityThe Tasmanian Study of Cognition and Gait. J. Gerontol. A Biol. Sci. Med. Sci. 75, 1537-1544. doi: 10.1093/gerona/glaa118

Lee, J. E., Park, H. J., Song, S. K., Sohn, Y. H., Lee, J. D., and Lee, P. H. (2010). Neuroanatomic basis of amnestic MCI differs in patients with and without Parkinson disease. Neurology 75, 2009-2016. doi: 10.1212/WNL. ob013e3181ff96bf

Litvan, I., Goldman, J. G., Tröster, A. I., Schmand, B. A., Weintraub, D., Petersen, R. C., et al. (2012). Diagnostic criteria for mild cognitive impairment in Parkinson's disease: Movement Disorder Society Task Force guidelines. Mov. Disord 27, 349-356. doi: 10.1002/mds.24893

Lo, O. Y., Halko, M. A., Zhou, J., Harrison, R., Lipsitz, L. A., and Manor, B. (2017). Gait Speed and Gait Variability Are Associated with Different Functional Brain Networks. Front. Aging Neurosci. 29:390. doi: 10.3389/fnagi.2017.00390

Mak, E., Su, L., Williams, G. B., Firbank, M. J., Lawson, R. A., Yarnall, A. J., et al. (2015). Baseline and longitudinal grey matter changes in newly diagnosed Parkinson's disease: ICICLE-PD study. Brain 138, 2974-2986. doi: 10.1093/ brain/awv211

Mc Ardle, R., Del Din, S., Galna, B., Thomas, A., and Rochester, L. (2020). Differentiating dementia disease subtypes with gait analysis: feasibility of wearable sensors? Gait Posture 76, 372-376. doi: 10.1016/j.gaitpost.2019.12.028

Mollenhauer, B., Rochester, L., Chen-Plotkin, A., and Brooks, D. (2014). What can biomarkers tell us about cognition in Parkinson's disease? Mov. Disord 29, 622-633. doi: 10.1002/mds.25846

Morris, R., Lord, S., Bunce, J., Burn, D., and Rochester, L. (2016). Gait and cognition: Mapping the global and discrete relationships in ageing and neurodegenerative disease. Neurosci. Biobehav. Rev. 64, 326-345. doi: 10.1016/ j.neubiorev.2016.02.012

Morris, R., Martini, D. N., Smulders, K., Kelly, V. E., Zabetian, C. P., Poston, K., et al. (2019). Cognitive associations with comprehensive gait and static balance measures in Parkinson's disease. Parkinsonism Relat. Disord. 69, 104-110. doi: 10.1016/j.parkreldis.2019.06.014

Nonnekes, J., Timmer, M. H., de Vries, N. M., Rascol, O., Helmich, R. C., and Bloem, B. R. (2016). Unmasking levodopa resistance in Parkinson's disease. Mov. Disord 31, 1602-1609. doi: 10.1002/mds.26712

Pereira, J. B., Aarsland, D., Ginestet, C. E., Lebedev, A. V., Wahlund, L. O., Simmons, A., et al. (2015). Aberrant cerebral network topology and mild cognitive impairment in early Parkinson's disease. Hum. Brain Mapp. 36, 2980-2995. doi: $10.1002 / \mathrm{hbm} .22822$

Plotnik, M., Giladi, N., Dagan, Y., and Hausdorff, J. M. (2011). Postural instability and fall risk in Parkinson's disease. Impaired dual tasking, pacing, and bilateral coordination of gait during the "ON" medication state. Exp. Brain Res. 210, 529-538. doi: 10.1007/s00221-011-2551-0

Postuma, R. B., Berg, D., Stern, M., Poewe, W., Olanow, C. W., Oertel, W., et al. (2015). MDS clinical diagnostic criteria for Parkinson's disease. Mov. Disord. 30, 1591-1601.

Ricciardi, C., Amboni, M., De Santis, C., and Ricciardelli, G. (2020). "Machine learning can detect the presence of Mild cognitive impairment in patients affected by Parkinson's Disease," in 2020 IEEE International Symposium on Medical Measurements and Applications, (MeMeA), 1-6.

Saredakis, D., Collins-Praino, L. E., Gutteridge, D. S., Stephan, B. C. M., and Keage, H. A. D. (2019). Conversion to MCI and dementia in Parkinson's disease: a systematic review and meta-analysis. Parkinsonism Relat. Disord 2019, 30215-30219. doi: 10.1016/j.parkreldis.2019.04.020

Siciliano, M., Santangelo, G., D’Iorio, A., Basile, G., Piscopo, F., Grossi, D., et al. (2016). Rouleau version of the Clock Drawing Test: age- and education-adjusted normative data from a wide Italian sample. Clin. Neuropsychol. 30, 1501-1516. doi: $10.1080 / 13854046.2016 .1241893$

Spinnler, H., and Tognoni, G. (1987). Standardizzazione e taratura italiana di una batteria di test neuropsicologici. Ital. J. Neurol. Sci. 8, 1-120.

Svenningsson, P., Westman, E., Ballard, C., and Aarsland, D. (2012). Cognitive impairment in patients with Parkinson's disease: diagnosis, biomarkers, and treatment. Lancet Neurol. 11, 697-707. doi: 10.1016/S1474-4422(12)7 0152-7

Xie, H., Wang, Y., Tao, S., Huang, S., Zhang, C., and Lv, Z. (2019). Wearable SensorBased Daily Life Walking Assessment of Gait for Distinguishing Individuals With Amnestic Mild Cognitive Impairment. Front. Aging Neurosci. 11:285. doi: $10.3389 /$ fnagi.2019.00285

Yildiz, D., Erer, S., Zarifoð̋lu, M., Hakyemez, B., Bakar, M., Karli, N., et al. (2015). Impaired cognitive performance and hippocampal atrophy in Parkinson disease. Turk. J. Med. Sci. 2015, 1173-1177.

Conflict of Interest: The authors declare that the research was conducted in the absence of any commercial or financial relationships that could be construed as a potential conflict of interest.

Publisher's Note: All claims expressed in this article are solely those of the authors and do not necessarily represent those of their affiliated organizations, or those of the publisher, the editors and the reviewers. Any product that may be evaluated in this article, or claim that may be made by its manufacturer, is not guaranteed or endorsed by the publisher.

Copyright (C) 2022 Amboni, Ricciardi, Cuoco, Donisi, Volzone, Ricciardelli, Pellecchia, Santangelo, Cesarelli and Barone. This is an open-access article distributed under the terms of the Creative Commons Attribution License (CC BY). The use, distribution or reproduction in other forums is permitted, provided the original author(s) and the copyright owner(s) are credited and that the original publication in this journal is cited, in accordance with accepted academic practice. No use, distribution or reproduction is permitted which does not comply with these terms. 